Research Article

\title{
Haemodynamic effects of intrathecal dexmedetomidine added to ropivacaine intraoperatively and for postoperative analgesia
}

\author{
Alka Shah*, Ila Patel, Rachana Gandhi
}

Department of Anaesthesiology, GMERS Medical College, Sola, Ahmedabad-380061, India

Received: 5 November 2012

Accepted: 11 December 2012

*Correspondence to:

Dr. Alka Shah,

Email: dralkap@yahoo.co.in

\begin{abstract}
Background: For lower abdomen and lower limb surgery, spinal anaesthesia is most common modality used in routine. This study was conducted on 50 ASA 1 and 2 planned for lower limb and lower abdomen surgery.

Methods: 50 patients of ASA 1 and 2 scheduled for lower limb and lower abdominal surgery were selected. Each patient received 4 milliliter volume of $0.75 \%$ isobaric ropivacaine +5 microgram dexmedetomidine. At the intervals of 1 minute, 2 minute, 5 minute, 10 minute, 20 minute, 30 minute and 1 hour, 2 hour and 3 hour reading of pulse rate and blood pressure were recorded. Postoperatively, pain scores were recorded by using Visual Analogue Scale.

Results: There were no significant changes in systolic and diastolic blood pressure after induction. The combination of ropivacaine and dexmedetomidine provided better postoperative analgesia and reduced requirement of diclofenac injection in first 24 hour.

Conclusions: The patients showed excellent hemodynamic stability and postoperative analgesia to ropivacaine + dexmedetomidine. Thus it is a safe modality for lower limb and lower abdomen surgery as far as haemodynamic effects and postoperative analgesia is concerned.
\end{abstract}

Keywords: Ropivacaine, Dexmedetomidine, Haemodynamic effects, Spinal block

\section{INTRODUCTION}

In the recent days regional techniques have come to take an upper hand in anaesthesia over general anaesthesia due to certain advantages like less chance of airway compromise and aspiration, facilitation of postoperative analgesia, benefit in some preexisting medical conditions and so on.

However most commonly drug used for spinal anaesthesia is bupivacaine, it's side effects like cardiotoxicity, neurotoxicity and more chances of hypotension has restricted it's use for certain cases. In such era luckily we have another safe option like ropivacaine which has reduced risk of cardiotoxicity, neurotoxicity and rapid recovery of motor function. ${ }^{1}$ However postoperative pain relief and haemodynamic stability are still an issue of concern.

Dexmedetomidine is highly selective alfa- 2 adrenergic agonists which reduces opioids and inhalational anaesthetic requirement and have been wildly used for Intensive Care Unit sedation with haemodynamic stability. Intrathecal alfa-2 receptor agonists have antinociceptive action for both somatic and visceral pain. ${ }^{2}$

We study the use of intrathecal combination of dexmedetomidine (5 microgram) with isobaric ropivacaine in lower limb surgeries. The aim of this study was to evaluate haemodynamic effects intraoperatively as well as to notify the duration of postoperative analgesia. The primary outcomes studied were hemodynamic parameter changes- especially blood pressure and pulse changes intraoperative as compare to preoperative. The duration of pain relief define as the time from intrathecal administration of ropivacaine + dexmedetomidine to first request for supplementary analgesia by the patients. Postoperative analgesia consumption in total and maximum have been evaluated as secondary outcome by pain score (Visual Analogue Scale).

\section{METHODS}

On arrival in the operating room patients were preloaded with lactated ringer's solution at 15 milliliter/kilogram. After obtaining written informed consent and approval from hospital ethical committee, 50 patients of ASA 1 
and 2 scheduled for lower limb and lower abdominal surgery were selected.

Patients who had contraindication to spinal anesthesia, allergy to drug and patients with heart block were excluded from the study. No premedication was given as it may interfere with assessment of hemodynamic data and drugs acting on heart rate and blood pressure were avoided preoperatively.

All patients were monitored with automated noninvasive blood pressure, pulse oximetry and electrocardiogram. A subarachnoid puncture was performed in sitting position at L3-4 or L4-5 interspace with 25 gauge quincke point spinal needle with all aseptic precautions. Following injection of isobaric ropivacaine $(0.75 \%) 4$ milliliter +5 microgram of dexmedetomidine, the patients were placed supine. Oxygen was given by mask if pulse oxymeter reading decreased below $90 \%$. At the intervals of 1 minute, 2 minute, 5 minute, 10 minute, 20 minute, 30 minute and 1 hour, 2 hour and 3 hour reading of pulse rate, blood pressure were recorded. Hypotension defined as a decrease in systolic blood pressure by more than $30 \%$ from baseline or less than 80 millimeter of mercury (systolic) was treated with intravenous ephedrine 5 milligram with boluses of fluid. Bradycardia defined as heart rate less than 50 beats per minute was treated with intravenous Atropine 0.6 milligram. The incidence of adverse effects such as nausea, vomiting, shivering, itching, pruritus, respiratory depression, sedation and hypotension was recorded. Sensory testing was assessed by loss of pin prick sensation to 23 gauge hypodermic needle at intervals of every 2 minutes till highest level had stabilized. Data regarding the highest dermatomal level of sensory blockade, the time to reach this level from the time of injection, time to $\mathrm{S} 2$ sensory regression and incidence of side effects were collected.

Postoperatively, pain scores were recorded by using Visual Analogue Scale between 0 and $10(0=$ no pain, 10 $=$ the most severe pain) initially every 1 hour for 2 hours, than every 2 hours for next eight hours and then after every 4 hours till 24 hours. Injection diclofenac 75 milligram intramuscular was given as rescue analgesia when Visual Analogue Score was 4 or more than 4. Follow up was carried out one week postoperatively for headache, pain and dysesthesias in buttock, thighs or lower limbs.

\section{RESULTS}

50 patients of ASA 1 and 2 scheduled for lower limb and lower abdominal surgery were selected. The patients in our study were belonged to age group 18-60 years. There was no significant difference in the mean age or weight or height and sex distribution among patients in our study.
Table 1 shows the changes in pulse rate after induction. There is no significant change occur in heart rate from baseline.

Table 1: Changes in heart rate from baseline.

\begin{tabular}{|ll|}
\hline Time & Mean \pm SD \\
\hline 1 minute (base line) & $94.4 \pm 2.15$ \\
\hline 2 minute & $98.5 \pm 4.16$ \\
\hline 5 minute & $88.44 \pm 4.52$ \\
\hline 10 minute & $85.96 \pm 1.71$ \\
\hline 20 minute & $83.12 \pm 2.10$ \\
\hline 30 minute & $82.60 \pm 1.97$ \\
\hline 1 hour & $80.64 \pm 3.05$ \\
\hline 2 hour & $82.20 \pm 3.50$ \\
\hline 3 hour & $82.12 \pm 5.15$ \\
\hline
\end{tabular}

Table 2 shows the changes in systolic blood pressure. There are no significant changes in systolic blood pressure after induction.

Table 2: Changes in systolic blood pressure.

\begin{tabular}{|ll|}
\hline Time & Mean \pm SD \\
\hline 1 minute (base line) & $111 \pm 5.74$ \\
\hline 2 minute & $110 \pm 4.10$ \\
\hline 5 minute & $98.24 \pm 4.74$ \\
\hline 10 minute & $94.08 \pm 5.80$ \\
\hline 20 minute & $100.24 \pm 4.37$ \\
\hline 30 minute & $103.2 \pm 4.32$ \\
\hline 1 hour & $103.3 \pm 2.32$ \\
\hline 2 hour & $105.33 \pm 4.42$ \\
\hline 3 hour & $106.56 \pm 3.80$ \\
\hline
\end{tabular}

Table 3 shows the changes in diastolic blood pressure. There are no significant changes in diastolic blood pressure after induction.

Table 3: Changes in diastolic blood pressure.

\begin{tabular}{|l|l|}
\hline Time & Mean \pm SD \\
\hline 1 minute (base line) & $74.56 \pm 6.12$ \\
\hline 2 minute & $72.4 \pm 3.1$ \\
\hline 5 minute & $68.8 \pm 5.80$ \\
\hline 10 minute & $65.36 \pm 4.92$ \\
\hline 20 minute & $66.56 \pm 3$ \\
\hline 30 minute & $69.56 \pm 3.89$ \\
\hline 1 hour & $70 \pm 4.86$ \\
\hline 2 hour & $71.36 \pm 3.72$ \\
\hline 3 hour & $72.8 \pm 4.35$ \\
\hline
\end{tabular}

Table 4 shows that combination of ropivacaine + dexmedetomidine provides better postoperative analgesia and reduces requirement of diclofenac injection in first 24 hour. 
Table 4: Summary of results.

\begin{tabular}{|ll|}
\hline & Mean \pm SD \\
\hline $\begin{array}{l}\text { Onset of sensory block } \\
\text { (minute) }\end{array}$ & $4.8 \pm 1.2$ \\
\hline $\begin{array}{l}\text { Time to achieve maximum } \\
\text { block (minute) }\end{array}$ & $11.7 \pm 1.7$ \\
\hline $\begin{array}{l}\text { Time of two segment } \\
\text { regression from highest } \\
\text { sensory level (minute) }\end{array}$ & $125.6 \pm 16.5$ \\
\hline $\begin{array}{l}\text { Time of regression to } \mathrm{S}_{2} \\
\text { (minute) }\end{array}$ & $468.3 \pm 36.8$ \\
\hline $\begin{array}{l}\text { Time of rescue analgesia } \\
\text { (minute) }\end{array}$ & $478.4 \pm 20.9$ \\
\hline $\begin{array}{l}\text { Highest pain score on visual } \\
\text { analogue score (0-10) }\end{array}$ & $4.4 \pm 1.4$ \\
\hline $\begin{array}{l}\text { Number of diclofenac } \\
\text { injection in first } 24 \text { hour } \\
\text { postoperatively }\end{array}$ & $0.97 \pm 0.19$ \\
\hline
\end{tabular}

As shown in table 5, the combination of ropivacaine + dexmedetomidine has negligible side effects.

Table 5: Side effects.

\begin{tabular}{|ll|}
\hline & No. \\
\hline Sedation & 0 \\
\hline Nausea & 1 \\
\hline Bradycardia & 1 \\
\hline Hypotension & 0 \\
\hline Shivering & 0 \\
\hline Respiratory depression & 0 \\
\hline
\end{tabular}

\section{DISCUSSION}

Various animal studies have been conducted in rats, rabbits, dogs and sheep using intrathecal dexmedetomidine at a dose range of 2.5 to 100 micrograms without any neurological deficit. ${ }^{3}$ Intrathecal dexmedetomidine in combination with bupivacaine have been studied in human being without any postoperative neurological deficits. ${ }^{4-8}$

Kalso et al reported that dexmedetomidine affinity to alfa-2 adrenoreceptor agonist is ten times as compared to clonidine. $^{2}$

De Kock et $\mathrm{al}^{9}$ who used clonidine with ropivacaine intrathecally in three different doses - 15, 45 and 75 microgram - for ambulatory knee arthroscopy, observed that small dose clonidine 15 microgram significantly improves the quality of anesthesia without delaying sensory and motor recovery, 45 microgram prolongs the sensory blockade without any influence on motor blockade but a dose of 75 microgram is associated with delayed sensory and motor recovery as well as detectable side effects as hypotension and sedation. From these studies, we had concluded that 5 microgram dexmedetomidine would be safe and appropriate for our study.

Al-Ghanem et $\mathrm{al}^{7}$ have reported the use of dexmedetomidine to be associated with a decrease in heart rate and blood pressure but in our study no cases of hypotension and bradycardia were noted. The reason could be combination of dexmedetomidine with ropivacaine has been shown to be a better drug in terms of cardiovascular and haemodynamic control. ${ }^{1}$

In our study, we found that the analgesic effect of intrathecal ropivacaine was potentiated by intrathecal dexmedetomidine. The addition of 5 microgram of intrathecal dexmedetomidine prolonged the postoperative analgesic effect of ropivacaine by approximately 8 hours. In addition, dexmedetomidine - treated group required less postoperative analgesic in the first 24 hours after surgery.

Intrathecal small dose of dexmedetomidine (3 microgram) used in combination with bupivacaine for spinal anaesthesia have been shown to produce a shorter onset of motor block and a prolongation in duration of motor and sensory block with haemodynamic stability shorter onset of motor block and a prolongation in duration of motor and sensory block with haemodynamic stability and lack of sedation. Al-Ghanem et al.'s study concluded that 5 microgram dexmedetomidine seems to be alternative as adjuvant to spinal bupivacaine in surgical procedures, especially in those who need quite long time with excellent quality of analgesia and minimal side effects. In our study we used intrathecal isobaric ropivacaine with dexmedetomidine for lower limb surgery.

The mechanism of action by which intrathecal alfa-2 adrenoceptor agonist prolong the motor and sensory block of local anaesthetics is not well known. The local anaesthetics act by blocking sodium channels, whereas the alfa-2 adrenoceptor agonist acts by binding to presynaptic C-fibres and post-synaptic dorsal horn neurons. The analgesic action of intrathecal alfa- 2 adrenoceptor agonist is by depressing the release of C-fibre transmitters and by hyperpolarisation of post-synaptic dorsal horn neurons. ${ }^{10}$ It may be an additive or synergistic effect secondary to the different mechanism of action of the local anaesthetic and the alfa-2 adrenoceptor agonist as studied by Salgado et al. ${ }^{11}$ This antinociceptive effect may explain the prolongation of sensory block when added to spinal anaesthetics. The prolongation of the motor block of spinal anaesthetics may result from the binding of alfa- 2 adrenoceptor agonists to motor neurons in the dorsal horn. ${ }^{12,13}$

The alfa-2 adrenergic agents also have antishivering property as observed by Talke et al. ${ }^{14}$, but we did not observe any incidence of it in our study. We also did not observe any haemodynamic side effect in our study. The 
reason may be, we had used small doses of intrathecal dexmedetomidine (5 microgram) in our study which was supported by Al-Ghanem et al. ${ }^{7}$

\section{CONCLUSION}

To conclude, 5 microgram dexmedetomidine seems to be an attractive alternative as an adjuvant to spinal ropivacaine in surgical procedures, especially those requiring long time. This combination (ropivacaine and dexmedetomidine) provides very good quality of haemodynamic stability. It has excellent quality of postoperative analgesia with minimal side effects. However, clinical studies to prove its efficacy and safety and varying dosages for supplementation of spinal local anaesthetics are recommended.

Funding: No funding sources Competing interests: None declared

Ethical approval: The study was approved by the hospital ethical committee

\section{REFERENCES}

1. McMamee DA, Convery PN, Milligan KR. Total knee replacement: a comparision of ropivacaine and bupivacaine in combine femoral and Sciatic block. Acta Anaesthesiol Scand 2001;45:477-81.

2. Kalso EA, Poyhia R, Rosenberg PH. Spinal antinociception by dexmedetomidine, a highly selective alfa-2 adrenergic agonist. Pharmacol Toxicol 1991;68:140-3.

3. Eisenach JC, Shafer SL, Bucklin BA, Jackson C, Kallio A. pharmacokinetics and pharmacodynamics of intraspinal dexmedetomidine in sheep. Anaesthesiology 1994;80:1349-59.

4. Fukushima K, Nishimi Y, Mori K, Takeda J. Effect of epidurally administered dexmedetomidine on sympathetic activity and postoperative pain in man. Anesth Analg 1996;82:S121.

5. Maroof M, Khan SA, Jain D, Khan RM, Maroof SM. Evaluation of effect of dexmedetomidine in reducing shivering following epidural anaesthesia. Anaesthesiology 2004;101:A495.

6. Kanazi GE, Aouad MT, Jabbour-Khoury SI, et al. Effect of low dose dexmedetomidine or clonidine on the characteristics of bupivacaine spinal block. Acta Anaesthesiol Scand 2006;50:222-7.

7. Al-Ghanem SM, Al-Mustafa MM, Al-Zaben KR, et al. Effects of adding dexmedetomidine versos fentanyl to intrathecal bupivacaine on spinal block characteristics in gynecological procedures. Am J Appl Sci 2009;6:882-7.

8. Al-Mustafa MM, Ammari BA, Awwad ZM, et al. Effects of dexmedetomidine added to spinal bupivacaine for urological procedure. Saudi Med J 2009;30:365-70.

9. De Kock M, Gautier P, Fanard L, et al. Intrathecal ropivacaine and clonidine for ambulatory knee arthroscopy. A dose response study. Anaesthesiology 2001;94:574-8.

10. Eisanach GC, De Kock M, Klimscha W. Alfa-2 adrenergic agonist for regional anaesthesia. Anaesthesiology 1996;85:655-74.

11. Salgado PF, Silva PC, Dalto HP, et al. Synergistic effect between dexmedetomidine and $0.75 \%$ ropivacaine in epidural anesthesia. Rev Assoc Med Bras 2008;54:110-5.

12. Harada Y, Nishioka K, Collins JG. Visceral antinociceptive effects of spinal clonidine combined with morphine, enkephalin, or U50, $488 \mathrm{H}$. Anaesthesiology 1995;83:344-52.

13. Yaksh TL, Reddy SV. Studies in primate on the analgesic effects associated with intrathecal actions of opiates, adrenergic agonists and baclofen. Anesthesiology 1981;54:451-67.

14. Talke P, Tayefeh F, Sessler DI, et al. Dexmedetomidine does not alter sweating threshold but comparably and linearly decreases the vasoconstriction and shivering thresholds. Anesthesiology 1997;87:835-41.

doi: 10.5455/2319-2003.ijbcp20130105

Cite this article as: Shah A, Patel I, Gandhi R.

Haemodynamic effects of intrathecal

dexmedetomidine added to ropivacaine

intraoperatively and for postoperative analgesia. Int $\mathrm{J}$

Basic Clin Pharmacol 2013;2:26-9. 